\title{
Time Protection in Operating Systems and Speaker Legitimacy Detection
}

\section{ADRIAN COLYER}

\section{OPERATING SYSTEM- BASED PROTECTION FROM TIMING-BASED SIDE-CHANNEL ATTACKS; IMPLICATIONS OF VOICE- IMITATION SOFTWARE}

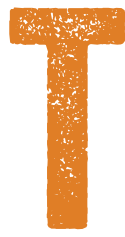

iming-based side-channel attacks are a particularly tricky class of attacks to deal with because the very thing you're of ten striving for-improved performance-can give you away. There are always more creative new instances of attacks to be found, so you need a principled way of thinking about defenses that address the class, not just a particular instantiation. That's what $\mathrm{Ge}$ et al. give us in "Time Protection, the Missing OS Abstraction." Just as operating systems prevent spatial inference through memory protection, so future operating systems will need to prevent temporal inference through time protection. It's going to be a long road to get there.

The second paper chosen for this edition comes from NDSS'19 (Network and Distributed System Security Symposium] and studies the physiological and social implications of the ever improving abilities of voiceimitation software. It seems people may be especially vulnerable to being fooled by fake voices. "The crux of voice (in)security: a brain study of speaker legitimacy detection," by Neupane et al., is a fascinating study with implications far beyond just the technology. 
For the complete column, go to https://queue.acm.org/TheMorningPaper/3/

Adrian Colyer is a venture partner with Accel in London, where it's his job to help find and build great technology companies across Europe and Israel. (If you're working on an interesting technology-related business, he would love to hear from you at acolyer@accel.com.] Prior to joining Accel, he spent more than 20 years in technical roles, including CTO at Pivotal, VMware, and SpringSource.

Copyright ( 2019 held by ownerlauthor. Publication rights licensed to ACM. Reprinted with permission from https:I/blog.acolyer.org. 